\title{
Lanthanum trichloride: an efficient Lewis acid catalyst for chemo and regioselective enamination of $\beta$-dicarbonyl compounds
}

\author{
R. Lenin and R. Madhusudhan Raju* \\ Department of Chemistry, Osmania University Hyderabad-500 007, India \\ E-mail: rallabaldimr@yahoo.co.in
}

\begin{abstract}
The various $\beta$-ketocarbonyl compounds rapidly form enaminone derivatives with a variety of amines at room temperature in the presence of a catalytic amount of lanthanum trichloride heptahydrate. The reaction proceeds smoothly at room temperature in methylene dichloride solvent.
\end{abstract}

Keywords: Dicarbonyl compounds, amines, enaminones, enamino esters, Lewis acid

\section{Introduction}

The enaminone derivatives are very important synthons for the synthesis of various heterocyclic and biologically active analogues including anti-inflammatory, ${ }^{1}$ antitumor, antibacterial, anticonvulsant agents, ${ }^{2}$ and intermediates for the synthesis of several amino acids, aminols, peptides and alkaloids. ${ }^{3} \quad$ Pyrroles, oxazoles, pyridinones, quinolines, dibenzodiazepines, tetrahydrobenzoxazines, tetronic acids, azasteroids, (1H)-pyridin-2-one, pyrazolo[1,5-a] pyrimidine and isoxazole derivatives have also been prepared from enaminones. Due to the wide range of utility in the pharmaceutical industry, the enamination of $\beta$-dicarbonyl compounds with various amines has become an important transformation and several methods have been developed for the synthesis of these compounds. Among them, the most simple and straightforward conventional method is the azeotropic removal of water by refluxing an amine and 1,3-diketone in aromatic solvents. ${ }^{4}$ The other methods used for this transformation with various activators such as Zinc perchlorate, $p$-toluene sulphonic acid, [EtNH$]_{3} \mathrm{NO}_{3}, \mathrm{CoCl}_{2}$, $\mathrm{InBr}_{3}, \mathrm{I}_{2}{ }^{5} \quad$ Sulphuric acid, $\mathrm{NaAuCl}_{4}, \mathrm{ZrCl}_{4}$, cerium chloride, $\mathrm{Sc}(\mathrm{OTf})_{3}$, alumina, silica; ${ }^{6}$ claymicrowave, ultrasound, sulfated zirconia, $\mathrm{Yb}(\mathrm{OTf})_{3}, \mathrm{TMSTf}^{7}$ acetic acid, $\mathrm{BF}_{3} . \mathrm{OEt}_{2}$, ionic liquid, $\mathrm{HClO}_{4}$, and solid supported solvent-free reactions have been reported. ${ }^{8}$ However, many of these methods have some drawbacks, which include expensive and stoichiometric amounts of reagents, suffer from poor regioselectivity and also require extended reaction times. Therefore, the development of a simple, more convenient and eco-friendly approaches is desirable. The 
catalyst lanthanum trichloride $\left(\mathrm{LaCl}_{3} .7 \mathrm{H}_{2} \mathrm{O}\right)$ heptahydrate is known for various organic transformations in the literature as a mild, inexpensive and moisture stable Lewis acid, ${ }^{9}$ and with an ever-increasing quest for new catalysts, herein, we report the use of $\mathrm{LaCl}_{3}$ as a novel and efficient catalyst for chemo and regioselective synthesis of $\beta$-enaminones and $\beta$-enaminoesters.

\section{Results and Discussion}

In a typical experiment, equimolar amounts of ethyl acetoacetate and benzyl amine in methylene dichloride solvent were stirred in the presence of lanthanum trichloride (10 mol\%) to obtain the corresponding 3-benzylamino-but-2-enoic acid ethyl ester in 93\% yield (entry a) and the reaction was completed within $3.0 \mathrm{~h}$ at room temperature. Encouraged by this result, the ethyl acetoacetate was treated with phenylethyl amine (entry b), butylamine (entry c) and aniline (entry d) to afford the corresponding products in excellent yields. In a similar manner, acetylacetone was reacted with various amines like benzylic (entry e) aliphatic (entry $\mathbf{f}, \mathbf{g}$ ) and aromatic (entry $\mathbf{h}$ ), to get the desired products in very good yields. In the case of another $\beta$-ketoester benzyl acetoacetate and various amines, the reactions takes place very smoothly to afford the desired products in very good yields (entry $\mathbf{i}, \mathbf{j}, \mathbf{k}, \mathbf{l}$ ). To confirm the role of catalyst, a blank reaction was carried out at similar reaction conditions with benzyl amine and ethylacetoacetate in absence of catalyst. But no progress was found even after stirring for a long reaction time (10h). It clearly shows the role of catalyst lanthanum trichloride for activation of the carbonyl group. All the reactions were completed within $3-5 \mathrm{~h}$ at room temperature to give the desired products in excellent yields (8593\%), with high regio and stereoselectivity, without forming any side products. In all the cases the catalyst was used in $10 \mathrm{~mol} \%$ only. In general the nucleophilic addition of amines to carbonyl compounds shows some steric effects on the rate of reaction. In the case of aliphatic amines the rate of reaction is little faster when compared with other amines. In a similar manner, the aromatic amines were a little slower than other amines under similar reaction conditions. All the products were identified by their ${ }^{1} \mathrm{H}$ NMR, IR and Mass spectroscopy data.

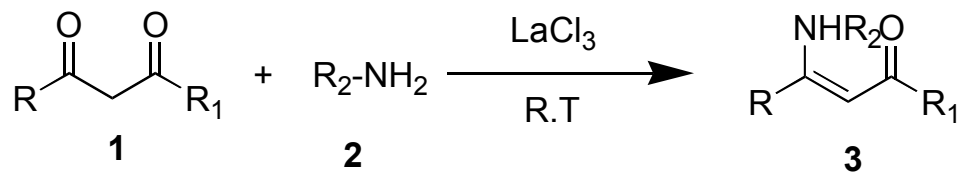

\section{Scheme 1}


Table 1. Lanthanum trichloride catalyzed enaminones synthesis

\begin{tabular}{|c|c|c|c|c|c|}
\hline Entry & Amine & Diketo compound & Product & $\begin{array}{l}\text { Reaction } \\
\text { Time (h) } \\
\end{array}$ & $\begin{array}{l}\text { Yield } \\
(\%)\end{array}$ \\
\hline $\mathrm{a}$ & & OEt & & 3.0 & 93 \\
\hline $\mathrm{b}$ & & & & 2.5 & 91 \\
\hline $\mathrm{c}$ & & & & 3.0 & 91 \\
\hline d & & & & 4.0 & 88 \\
\hline e & & & & 3.5 & 92 \\
\hline $\mathrm{f}$ & & & & 3.0 & 90 \\
\hline $\mathrm{g}$ & & & & 3.5 & 89 \\
\hline $\mathrm{h}$ & & & & 4.5 & 86 \\
\hline i & & $n$ & & 4.0 & 92 \\
\hline J & & $\mathrm{OBn}$ & & 3.5 & 90 \\
\hline $\mathrm{k}$ & & OBn & & 4.0 & 89 \\
\hline 1 & & & & 5.0 & 85 \\
\hline
\end{tabular}




\section{Conclusions}

In conclusion, lanthanum trichloride was found to be an efficient catalyst for the synthesis of $\beta$ enaminones and $\beta$-enaminoesters in very good to excellent yields. This methodology offers several advantages including mild reaction conditions, enhanced reaction rates, easy isolation of products and operational simplicity. The above merits make this protocol superior to existing methodologies for the synthesis of $\beta$-enaminones and $\beta$-enaminoesters.

\section{Experimental Section}

General Methods. Melting points were recorded on Buchi R-535 apparatus and are uncorrected. I.R spectra were recorded on a Perkin-Elmer FT-IR 240-c Spectro photometer. ${ }^{1}$ HNMR spectra were recorded on Gemini-200 spectrometer in $\mathrm{CDCl}_{3}$ using TMS as internal standard. Mass spectra were recorded on a Finnigan MAT 1020 mass spectrometer operating at $70 \mathrm{eV}$. All the products physical and spectroscopic data were compared with those reported in the literature.

General experimental procedure. To a mixture of 1, 3-diketoester compound (2.0 mmol) and benzylamine $(2.0 \mathrm{mmol})$ in methylenedichloride $(10 \mathrm{ml})$ was added the lanthanum trichloride $(0.2 \mathrm{mmol})$. The resulting reaction mixture was stirred at room temperature for a specified period (see table 1). The completion of the reaction was confirmed by thin layer chromatography. Then the reaction mixture was diluted by adding DCM $(20 \mathrm{ml})$ and washed with water, followed by brine solution. The organic layer was dried over $\mathrm{Na}_{2} \mathrm{SO}_{4}$ and concentrated under reduced pressure. The obtained crude products were purified by column chromatography, using silica gel 60-120 mesh. The obtained pure products were confirmed by their ${ }^{1} \mathrm{H}$ NMR, IR and mass spectroscopy.

\section{Spectral data for selected compounds}

3-Benzylamino-but-2-enoic acid ethyl ester. (3a).Colorless oil. IR (neat): v 3294, 3041, 2971, $2839,1628,1605,1561,1456,1375,1281,1231,1169,1057,1012,978,846,785,741 \mathrm{~cm}^{-1} .{ }^{1} \mathrm{H}$ $\operatorname{NMR}\left(\mathrm{CDCl}_{3}\right): \delta 1.28(\mathrm{t}, 3 \mathrm{H}, J=7.0 \mathrm{~Hz}), 1.90(\mathrm{~s}, 3 \mathrm{H}), 4.10(\mathrm{q}, 2 \mathrm{H}, J=7.0 \mathrm{~Hz}), 4.42(\mathrm{~d}, 2 \mathrm{H}, J=$ $5.0 \mathrm{~Hz}$ ), 4.50 (s, 1H), 7.20-7.40 (m, 5H), 8.98 (brs, 1H, NH). EIMS: m/z (\%): 219 (m $\left.{ }^{+} 25\right), 178$ (18), 134 (100), 106 (31), 77 (56), 52 (15).

3-Butylamino-but-2-enoic acid ethyl ester. (3c). Light yellow oil. IR (neat): $v$ 3145, 3018, $1641,1608,1583,1406,1367,1273,1210,1185,1061,1006,961,843,755,732 \mathrm{~cm}^{-1}$. ${ }^{1} \mathrm{H}$ NMR $\left(\mathrm{CDCl}_{3}\right): \delta 0.98(\mathrm{t}, 3 \mathrm{H}, J=6.8 \mathrm{~Hz}), 1.27(\mathrm{t}, 5 \mathrm{H}, J=6.8 \mathrm{~Hz}), 1.40-1.48(\mathrm{~m}, 1 \mathrm{H}), 1.52-1.62(\mathrm{~m}$, $1 \mathrm{H}), 1.92(\mathrm{~s}, 3 \mathrm{H}), 3.20(\mathrm{q}, 2 \mathrm{H}, J=7.0 \mathrm{~Hz}), 4.05(\mathrm{~d}, 2 \mathrm{H}, J=5.0 \mathrm{~Hz}), 4.38(\mathrm{~s}, 1 \mathrm{H}), 8.55$ (brs, $1 \mathrm{H}$, NH). EIMS: $m / z$ (\%): 185 (m $\left.{ }^{+} 100\right), 153$ (10), 140 (10), 96 (46), 71 (10), 67 (20), 55 (50), 43 (45). 
4-Phenylamino-pent-3-en-2-one (3h). Solid. mp, 48-49 ${ }^{0} \mathrm{C}$ IR (neat). v 3453, 3137, 3028, 2974 , 2846, 1622, 1576, 1510, 1461, 1329, 1274, 1205, 1187, 1031, 921, 856, $749 \mathrm{~cm}^{-1}$. ${ }^{1} \mathrm{H}$ NMR $\left(\mathrm{CDCl}_{3}\right) . \delta 0.97(\mathrm{t}, 3 \mathrm{H}, J=6.8 \mathrm{~Hz}), 1.90(\mathrm{~s}, 3 \mathrm{H}), 4.95(\mathrm{~s}, 1 \mathrm{H}), 7.20-7.40(\mathrm{~m}, 5 \mathrm{H}), 8.98(\mathrm{brs}, 1 \mathrm{H}$, NH). EIMS: $m / z$ (\%). $175\left(\mathrm{~m}^{+} 10\right), 156$ (15), 140 (25), 110 (10), 96 (100), 84 (30), 71 (26), 56 (22), 43 (78).

3-Phenylamino-but-2-enoic acid benzyl ester.(3I). IR (neat): $v$ 3256, 3071, 2954, 2832, 1629, $1602,1573,1504,1429,1362,1251,1249,1163,1057,1005,978,863,739 \mathrm{~cm}^{-1}$. ${ }^{1} \mathrm{H}$ NMR $\left(\mathrm{CDCl}_{3}\right): \delta 1.92(\mathrm{~s}, 3 \mathrm{H}), 4.42(\mathrm{~s}, 1 \mathrm{H}), 5.08(\mathrm{~s}, 2 \mathrm{H}), 5.16(\mathrm{~s}, 2 \mathrm{H}), 7.25-7.40(\mathrm{~m}, 10 \mathrm{H}), 8.98$ (brs, 1H, NH). EIMS: m/z (\%). $281\left(\mathrm{~m}^{+} 10\right), 217$ (10), 208 (10), 193 (12), 175 (100), 168 (30), 150 (80), 81 (75), 79 (55), 67 (18), 52 (15), 43 (100).

\section{References and Notes}

1. (a) Foster, J. E.; Nicholson, J. M.; Butcher, R.; Stables, J. P.; Edafiogho, I. O.; Goodwin, A. M.; Henson, M. C.; Smith, C. A.; Scott, K. R. Bioorg. Med. Chem. 1999, 7, 2415. (b) Michael, J. P.; Koning, C. B.; Hosken, G. D.; Stanbury, T. V. Tetrahedron 2001, 57, 9635. (c) Spivey, A. C.; Srikaran, R.; Diaper, C. M.; Turner, D. J. Org. Biomol. Chem. 2003, 1, 1638. (d) Dannhardt, G.; Bauer, U.; Nowe, J. J. Prakt. Chem. 1998, 340, 256.

2. (a) Boger, D. L.; Ishizaki, T.; Wysocki, J. R. J.; Munk, S. A.; Kitos, P. A.; Suntornwar, O. J. Am. Chem. Soc. 1989, 111, 6461. (b) Wang, Y. F.; Izawa, T.; Kobayashi, S.; Ohno, M. J. Am. Chem. Soc. 1982, 104, 6465. (c) Natalie, D. E.; Donna, S. C.; Khurana, M.; Noha, N. S.; James, P. S.; Sylvia, J. H.; Abraham, N.; Robert, S. T.; Jacqueline, A. M. Eur. J. Med. Chem. 2003, 49.

3. (a) Potin, D.; Dumas, F.; d'Angelo, J. J. Am. Chem. Soc. 1990, 112, 3483. (b) Bartoli, G.; Cimarelli, C.; Marcantoni, E.; Palmieri, G.; Petrini, M. J. Org. Chem. 1994, 59, 5328. (c) Palmieri, G.; Cimmerelli, C. J. Org. Chem. 1996, 61, 5557. (d) Beholz, L. G.; Benovsky, R.; Ward, D. L.; Bata, N. S.; Stille, J. R. J. Org. Chem. 1997, 62, 1033. (e) Ferraz, H. M. C.; Pereira, F. L. C.; Leite, F. S.; Nuns, M. R. S.; Payretarrua, M. E. Tetrahedron. 1999, 55, 10915. (f) David, O.; Blot, J.; Bellec, C.; Bellassoued, M. C. F.; Haviari, G.; Celerier, J. P.; Lhommet, G.; Gramain, J. C.; Garadette, D. J. Org. Chem. 1999, 64, 3122.

4. (a) Baraldi, P. G.; Simoni, D.; Manfredini, S. Synthesis. 1983, 902. (b) Corey, E. J.; Garner, R. H.; Hayes, A. W. J. Org. Chem. 1972, 37, 4436.

5. (a) Zhang, Z. H.; Yin, L.; Wand, Y. M. Adv. Synth. Catal. 2006, 348, 184. (b) Bartoli, G.; Bosco, M.; Locatelli, M.; Marcantoni, E.; Melchiorre, P.; Sambri, L. Synlett 2004, 239. (c) Hickmott, P. W.; Sheppard, G. J. Chem. Soc., Perkin Trans. 1 1972, 1038. (d) Hauser, C. R.; Reynolds, G. A. J. Am. Chem. Soc. 1948, 70, 2402. (e) Zhang, Z.-H.; Hu, J. -Y.; J. Braz. Chem. Soc. 2006, 17, 1447. (f) Bhosale, R. S.; Suryawanshi, P. A.: Ingle, S. A.; Lokhande, M. N.; More, S. P.; Mane, S. B.; Bhosale, S. V.; Pawar, R. P.; Synlett, 2006, 933. 
6. (a) Martin, D. F.; Janusonis, G. A.; Martin, B. B. J. Am. Chem. Soc. 1961, 83, 73. (b) Arcadi, A.; Bianchi, G.; Di Giuseppe, S.; Marinelli, F. Green Chem. 2003, 5, 64. (c) Khodaei, M. M.; Khosropour, A. R.; Kookhazadeh, M. Synlett 2004, 1980. (d) TexierBouliet. T. Synthesis, 1985, 679. (e) Gao, Y. H.; Zhang, Q. H.; Xu, J. X. Synth. Commun. 2004, 34, 909. (f) Yadav, J. S.; Kumar, V. N.; Rao, R. S.; Priya, D. A. D.; Rao, P. P.; Reddy, B. V. S.; Nagaiah, K. J. Mol. Catal. A: Chem. 2006, 256, 243. (g) Lin, J.; Zhang, L. F. Monatsh. Chem. 2007, 138, 77.

7. (a) Cartaya-Martin, C. P.; Henderson, D. G.; Soeder, R. W. Synth. Commun. 1997, 4275. (b) Rechsteimer, B.; Texier-Boullet, F.; Hamelin, J. Tetrahedron Lett. 1993, 34, 5074. (c) Silva, F. C.; De Souza, M. C. B. V.; Ferreira, V. F.; Sabino, S. J.; Antunes, O. A. C. Catal. Commun. 2004, 5, 151. (d) Valduga, C. J.; Squizani, A.; Braibante, H. S.; Braibante, M. E. F. Synthesis 1998, 1019. (e) Zhang, Z.-H.; Song, L. -M.; J. Chem. Res. 2006, 817. (f) Varala, R.; Nuvula, S.; Adapa, S. R. Aust. J. Chem. 2006, 59, 921.

8. (a) Stefane, B.; Polanc, S. Synlett 2004, 698. (b) Silva, F. C.; De Souza, M. C. B. V.; Ferreira, V. F.; Sabino, S. J.; Antunes, O. A. C. Synthesis 2004, 1557. (c) Gholap, A. R.; Chakor, N. S.; Daniel, T.; Lahoti, R. J.: Srinivasan, K. V. J. Mol. Catal A. 2006, $245,37$. (d) Das, B.; Lu, K. V.; Majhi, A.; Reddy, M. R.; Reddy, K. N.; Rao, Y. K.; Kumar, K. R.; Sridhar, B. J. Mol. Catal. A:, Chem. 2006, 246, 276. (e) Karhikeyan, G.; Perumal, T.; Can. J. Chem. 2005, 1746.

9. (a) Luche, J. L.; Gemal, A. L. Chem. Commun. 1978, 976. (b) Narsaiah, A, V.: Nagaiah, K. Indian J. Chem., 2003, 2045. (c) Narsaiah, A, V.: Nagaiah, K. Synth. Commun. 2003, 3825.

10. The used catalyst Lanthanum trichloride heptahydrate $\left(\mathrm{LaCl}_{3} .7 \mathrm{H}_{2} \mathrm{O}\right)$ was supplied from Sigma-Aldrich group. 